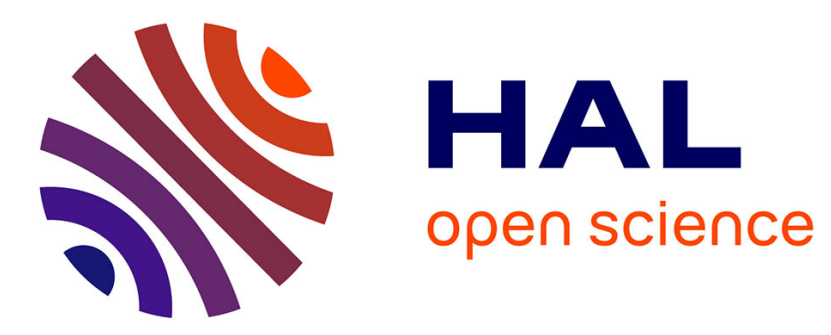

\title{
Measuring influence among players with an ordered set of possible actions
}

\author{
Michel Grabisch, Agnieszka Rusinowska
}

\section{To cite this version:}

Michel Grabisch, Agnieszka Rusinowska. Measuring influence among players with an ordered set of possible actions. 2008. halshs-00260863

\section{HAL Id: halshs-00260863 \\ https://shs.hal.science/halshs-00260863}

Submitted on 5 Mar 2008

HAL is a multi-disciplinary open access archive for the deposit and dissemination of scientific research documents, whether they are published or not. The documents may come from teaching and research institutions in France or abroad, or from public or private research centers.
L'archive ouverte pluridisciplinaire HAL, est destinée au dépôt et à la diffusion de documents scientifiques de niveau recherche, publiés ou non, émanant des établissements d'enseignement et de recherche français ou étrangers, des laboratoires publics ou privés. 
Measuring influence among players

with an ordered set of possible actions

Michel Grabisch, Agnieska Rusinowska

Janvier 2008

GATE Groupe d'Analyse et de Théorie Économique UMR 5824 du CNRS

93 chemin des Mouilles - 69130 Écully - France

B.P. 167 - 69131 Écully Cedex

Tél. +33 (0)4 72866060 - Fax +33 (0)4 72866090

Messagerie électronique gate@gate.cnrs.fr

Serveur Web : www.gate.cnrs.fr 


\title{
Measuring influence among players with an ordered set of possible actions
}

\author{
MICHEL GRABISCH${ }^{1}$ and AGNIESZKA RUSINOWSKA ${ }^{2}$ \\ 1 Université Paris I Panthéon-Sorbonne \\ CERMSEM, 106-112 Bd de l'Hôpital, 75013 Paris, France \\ Michel.Grabisch@lip6.fr \\ 2 GATE, CNRS UMR 5824 - Université Lumière Lyon 2 - Ecole Normale Supérieure LSH \\ 93, Chemin des Mouilles - B.P.167, 69131 Ecully Cedex, France \\ rusinowska@gate.cnrs.fr
}

\begin{abstract}
In the paper, we introduce and study generalized weighted influence indices of a coalition on a player, where players have an ordered set of possible actions. Each player has an inclination to choose one of the actions. Due to influence of a coalition of other players, a final decision of the player may be different from his original inclination. An influence in such situations is measured by the general weighted influence index. In a particular case, the decision of the player may be closer to the inclination of the influencing coalition than his inclination was. The weighted influence index which captures such a case is called the positive weighted influence index. We also consider the negative weighted influence index, where a final decision of the player goes farther away from the inclination of the influencing coalition. Some special cases of the weighted influence indices, called a possibility influence index and an equidistributed influence index, are also defined. We consider different influence functions and study their properties. A set of followers and a set of a conditional followers of a given coalition are defined, and their properties are analyzed. We define the concepts of success, decisiveness, luck, and failure for the multi-choice model of influence.
\end{abstract}

\section{JEL Classification: C7, D7}

Keywords: general weighted influence index, positive weighted influence index, negative weighted influence index, influence function, follower of a coalition, success, decisiveness

\section{Introduction}

The point of departure of this paper is the concept of decisional power (the Hoede-Bakker index) which has been proposed in [4], and later generalized and modified in [8]. In the original framework, we consider a social network with players, also referred to as agents or actors, who have to make an acceptance-rejection decision. In the original framework it is assumed that each player has an inclination to say 'yes' or 'no'. Then, each player makes his yes-no decision which, due to influence of other players, may be different from the original inclination of the player. Furthermore, a group decision is made. If we define a success of a player as a probability that the player is successful, and a successful player is defined as a player whose inclination coincides with the group decision, then we get the following interpretation of the Hoede-Bakker index. If all inclination vectors are equally probable, then the generalized Hoede-Bakker index is a kind of a 'net' Success (see [7]), and moreover, the generalized Hoede-Bakker index coincides with the absolute Banzhaf index; see [8]. Although the influence aspects incorporated into the social network make the index especially attractive, both from theoretical and application point of view, there is an essential drawback of the (generalized) Hoede-Bakker index. The main drawback of 
the index is that the actual role of the influence function is hidden. In [3], a general form of the index is proposed, called a global index, which enables the analysis of influence among players. The global index is defined as a pair consisting of an influence index and the revised Hoede-Bakker index. The authors define weighted influence indices and study their properties. Several special cases of the weighted influence indices are considered, like the possibility influence index, and the certainty influence index. Two kinds of influence, a direct influence and an opposite influence, are studied. Under the direct influence, the influencing coalition succeeds in making a player vote according to the inclination of the coalition, while the inclination of the player was different from the inclination of the coalition. The opposite influence of a coalition on a player means that the inclinations of the player and the coalition coincide, but the player's vote is different from this inclination. The aim of the present paper is to enlarge the set of possible yes-no decisions to multichoice games (see e.g., [5], and also [2]), and to investigate the generalized influence indices. In fact, we escape now from a voting situation, where players have to say either 'yes' or 'no'. It is assumed that each player has a totally ordered set of possible actions, the same for each player, and he has to choose one of the actions. A player has an inclination to choose a particular action, but his decision may be different from the inclination, due to the influence of other players. There is no outside event which could make a player change his decision: only interactions between players take place. Consequently, if a player decides differently from his original inclination, it may be seen as a unique result of the influence between players. First, we propose a general form of the influence index for the new framework, in which players have an ordered set of possible actions. The general weighted (respectively general possibility) influence index is a natural generalization of the weighted (respectively possibility) direct influence index defined for yes-no scheme in [3]. Next, we analyze a positive influence, which measures how much a coalition attracts a player, i.e., pulls the player's vote near to the inclination of the coalition. A player who has an inclination different from the inclination of a given coalition is influenced by this coalition if his (final) decision is closer to the inclination of the coalition than his (original) inclination was. We also investigate a negative influence. For each inclination vector in which the members of a given coalition have the same inclination, there is one (or two) action(s) which is (are) the most extreme action(s). These actions lie 'farthest' from the inclination of the coalition. If the inclination of a player is different from the most extreme action of the coalition, and his decision comes 'closer' to the extreme action, we say that there is the negative influence of the coalition on the player. An opposite influence in the yes-no model, which was investigated in [3], is a particular case of the negative influence: the inclinations of the player and the coalition are the same, but the decision of the player is different from the inclination of the coalition. When all actors make their final decisions, a group decision is determined. Similar as in the original framework, we do not restrict our analysis to one particular group decision function, but we allow for different group decision functions.

In the set of possible actions, we display a set of 'neutral actions', which are defined such that a coalition with the inclination to choose the neutral action has no ability to influence a player. Hence, if a player decides differently from his inclination, it is assumed to be NOT due to the influence of a coalition with the 'neutral inclination'. One particular case of the three-action model is a generalization of the original yes-no framework to a yes-no-abstention scheme (see [2]), where each player has an inclination to say either 'yes' 
(action +1 ), or 'no' (action -1 ), or to abstain (action 0 ). The abstention may be defined as such a neutral action. It is then assumed that a coalition of abstaining players has no influence ability, but the abstention may be a result of the influence.

In the paper, we consider the set of followers, where by a follower of a given coalition of players we mean the agent who always decides according to the inclination of the coalition in question. We also define the set of conditional followers, i.e., players who follow a given coalition only if the coalition has an inclination to choose one particular action. Moreover, different influence functions are defined and analyzed.

The structure of the paper is the following. In Section 2 the generalized influence indices are defined and studied. For simplicity reasons we do not add the word generalized when defining and discussing the indices, but they all measure an influence in a more general framework, and they are a generalized version of the ones considered in [3]. In Section 3, we introduce the concepts of a follower of a coalition, a conditional follower, and a purely influential function. Different influence functions and their properties are investigated in Section 4. The notions of success, decisiveness, and related concepts are considered in Section 5. In Section 6 we conclude. We also present several examples.

\section{The generalized influence indices}

The general framework is the following. We consider a social network with the set of players (agents, actors) denoted by $N=\{1, \ldots, n\}$. There is a totally ordered set of possible actions $A$, where $\infty>|A| \geq 2$. For simplicity we assume each action to be an integer. Each player has an inclination to choose one of the actions. Hence, by the inclination of a player we mean the action the player wants to choose. An inclination vector, denoted by $i$, is an $n$-vector consisting of the actions of the players. Let $I$ be the set of all inclination vectors, $I=A^{n}$. It is assumed that players may influence each others, and due to the influences in the network, the final decision of a player may be different from his original inclination. In other words, each inclination vector $i \in I$ is transformed into a decision vector $B i$, where $B: I \rightarrow C^{n}$ is the influence function, and $C \subseteq A$ is the set of possible decisions. The set of all influence functions will be denoted by $\mathcal{B}$. The decision vector $B i$ is an $n$-vector consisting of the decisions made by the players. Furthermore, given $G \subseteq A$, the group decision function $g d: C^{n} \rightarrow G$ is introduced, having the value $a \in G$ if the group decision is to choose the action $a$. The set of all group decision functions will be denoted by $\mathcal{G}$. By assuming $C^{n}$ instead of $I$ in the influence function, and $G \subseteq A$ instead of $A$ in the group decision function, we allow for the possibility that not all actions in $A$ can be chosen as the decision of a player and as the group decision. For instance, if the actions in $A$ are interpreted as degrees of inclination, which are between NO (denoted by -1 ) , ..., 'maybe NOT', ..., 'maybe YES', and YES (denoted by +1), then both the decision (interpreted as the vote) and the group decision could be just one of the two actions: either NO or YES.

In the set of all actions $A$, we distinguish the subset $A^{0} \subset A$, possibly empty, which is the set of all neutral actions. A neutral action is defined as an action such that a coalition with the inclination to choose the neutral action has no ability to influence a player. On the other hand, a neutral action may be a result of the influence.

Remark 1 One can see immediately our model as a natural generalization of the original yes-no model, which we simply define as $A=\{-1,+1\}$ and $A^{0}=\emptyset$. Moreover, we capture 
a yes-no-abstention model by setting $A=\{-1,0,+1\}$ and $A^{0}=\{0\}$ if we assume that an abstaining coalition has no ability to influence. On the other hand, if a coalition with the inclination to abstain is assumed to be able to influence a player, we can simply consider a three-action model with $A=\{-1,0,+1\}$ and $A^{0}=\emptyset$.

Let us focus on the influence issue. Before formalizing the influence concepts, we introduce several notations for convenience. First of all, cardinality of sets $S, T, \ldots$ will be denoted by the corresponding lower case $s, t, \ldots$ We omit braces for sets, e.g., $\{k, m\}$, $N \backslash\{j\}, S \cup\{j\}$ will be written $k m, N \backslash j, S \cup j$, etc.

We introduce for any $\emptyset \neq S \subseteq N$ the set

$$
I_{S}:=\left\{i \in I \mid \forall k, j \in S\left[i_{k}=i_{j} \wedge i_{k} \in A \backslash A^{0}\right]\right\} .
$$

We denote by $i_{S} \in A \backslash A^{0}$ the value $i_{k}$ for some $k \in S, i \in I_{S}$.

The concept of the influence adopted in Subsections 2.1 and 2.2 is related to the concept of the direct influence of a coalition on a player defined in [3]. It is assumed that the player's inclination is different from the inclination of the coalition, and the player's decision differs from his inclination. Let for each $S \subseteq N$ and $j \in N \backslash S$

$$
\begin{gathered}
I_{S \rightarrow j}:=\left\{i \in I_{S} \mid i_{j} \neq i_{S}\right\} \\
I_{S \rightarrow j}^{*}(B):=\left\{i \in I_{S \rightarrow j} \mid(B i)_{j} \neq i_{j}\right\} .
\end{gathered}
$$

$I_{S \rightarrow j}$ and $I_{S \rightarrow j}^{*}(B)$ denote the set of all inclination vectors of potential influence of $S$ on $j$, and the set of all inclination vectors of observed influence of $S$ on $j$ under given $B \in \mathcal{B}$, respectively.

\subsection{The general influence indices}

First, we can define the weighted influence indices as they were defined for the yes-no model in [3]. We will call them the general influence indices. For each $S \subseteq N, j \in N \backslash S$ and $i \in I_{S \rightarrow j}$, we introduce a weight $\alpha_{i}^{S \rightarrow j} \in[0,1]$ of influence of coalition $S$ on $j \in N \backslash S$ under the inclination vector $i \in I_{S \rightarrow j}$. We assume that for each $S \subseteq N$ and $j \in N \backslash S$, there exists $i \in I_{S \rightarrow j}$ such that $\alpha_{i}^{S \rightarrow j}>0$. Moreover, we impose a kind of the symmetry assumption that $\alpha_{i}^{S \rightarrow j}$ depends solely on numbers $n^{a}(S, j, i)$, for $a \in A \backslash\left(A^{0} \cup\left\{i_{j}\right\}\right)$, where $n^{a}(S, j, i)$ is the number of players with the inclination to choose action $a \in A \backslash\left(A^{0} \cup\left\{i_{j}\right\}\right)$ under $i \in I_{S \rightarrow j}$, i.e., for each $S \subseteq N, j \notin S, i \in I_{S \rightarrow j}$, and $a \in A \backslash\left(A^{0} \cup\left\{i_{j}\right\}\right)$,

$$
n^{a}(S, j, i):=\left|\left\{m \in N \backslash j \mid i_{m}=a\right\}\right|
$$

Definition 1 Given $B \in \mathcal{B}$, for each $S \subseteq N, j \in N \backslash S$, the general weighted influence index of coalition $S$ on player $j$ is defined as

$$
d_{\alpha}(B, S \rightarrow j):=\frac{\sum_{i \in I_{S \rightarrow j}^{*}(B)} \alpha_{i}^{S \rightarrow j}}{\sum_{i \in I_{S \rightarrow j}} \alpha_{i}^{S \rightarrow j}} \in[0,1] .
$$

Moreover, the general possibility influence index of coalition $S$ on player $j$ is given by

$$
\bar{d}(B, S \rightarrow j)=d_{\bar{\alpha}}(B, S \rightarrow j)
$$




$$
\text { where } \bar{\alpha}_{i}^{S \rightarrow j}=1 \text { for each } i \in I_{S \rightarrow j} \text {, }
$$

and the general equidistributed influence index of coalition $S$ on player $j$ is given by

$$
\begin{gathered}
d^{*}(B, S \rightarrow j)=d_{\alpha^{*}}(B, S \rightarrow j) \\
\text { where } \alpha_{i}^{* S \rightarrow j}=\frac{1}{\sum_{a \in A \backslash\left(A^{0} \cup\left\{i_{j}\right\}\right)}\left(2^{n^{a}(S, j, i)}-1\right)} \text { for each } i \in I_{S \rightarrow j} .
\end{gathered}
$$

Note that the general possibility influence index of coalition $S$ on player $j$ is then equal to

$$
\bar{d}(B, S \rightarrow j):=\frac{\left|I_{S \rightarrow j}^{*}(B)\right|}{\left|I_{S \rightarrow j}\right|} \in[0,1] .
$$

For the equidistributed influence index, for each $S \subseteq N, j \in N \backslash S, i \in I_{S \rightarrow j}$, and for each $a \in A \backslash\left(A^{0} \cup\left\{i_{j}\right\}\right)$ we count the number of all $p$-player coalitions of the set $\left\{m \in N \backslash j \mid i_{m}=a\right\}$, where $p=1, \ldots, n^{a}(S, j, i)$, which gives

$$
\sum_{p=1}^{n^{a}(S, j, i)}\left(\begin{array}{c}
n^{a}(S, j, i) \\
p
\end{array}\right)=2^{n^{a}(S, j, i)}-1 .
$$

As one can see, players having either the same inclination as the influenced player or the inclination to choose a neutral action, are not considered as responsible for the influence.

\subsection{The positive influence indices}

The concept of the general influence index seems to be sufficient to register just the fact of the influence between players, since it considers all cases when a player's decision is different from his inclination. Nevertheless, the general influence index does not distinguish 'how much' a player changes his 'position'. In the yes-no framework, if a player votes differently than his inclination was, the change is defined uniquely, since there are only two possibilities: 'yes' or 'no'. In the model with an ordered set of possible actions, we can observe more than just the changes, i.e., we have also an information about different distances between actions. Consequently, in the generalized model with an ordered set of possible actions, we need to introduce a more sophisticated measure of the influence. Given coalition $S \subset N$, player $j \notin S$, and inclination vector $i \in I_{S \rightarrow j}$, there is a certain (positive) distance $\left|i_{j}-i_{S}\right|$ between $i_{j}$ and $i_{S}$. Under the influence, the decision $(B i)_{j}$ of player $j$ may be different from his inclination, and what we can also measure is a distance $\left|(B i)_{j}-i_{S}\right|$ between the decision of the player and the inclination of the coalition. Did the coalition succeed in making the player 'put' his decision closer to the inclination of the coalition? Consequently, for each $S \subseteq N, j \in N \backslash S$, and $B \in \mathcal{B}$, we can define the set of all inclination vectors of positive influence as

$$
I_{S \rightarrow j}^{\text {pos }}(B):=\left\{i \in I_{S \rightarrow j}||(B i)_{j}-i_{S}|\leq| i_{j}-i_{S} \mid\right\} .
$$

Definition 2 Given $B \in \mathcal{B}$, for each $S \subseteq N, j \in N \backslash S$, the positive weighted influence index of coalition $S$ on player $j$ is defined as

$$
D_{\alpha}(B, S \rightarrow j):=\frac{\sum_{i \in I_{S \rightarrow j}^{\text {pos }}(B)}\left[\left|i_{j}-i_{S}\right|-\left|(B i)_{j}-i_{S}\right|\right] \alpha_{i}^{S \rightarrow j}}{\sum_{i \in I_{S \rightarrow j}}\left|i_{j}-i_{S}\right| \alpha_{i}^{S \rightarrow j}} \in[0,1] .
$$


The positive possibility influence index of coalition $S$ on player $j$ is given by

$$
\begin{gathered}
\bar{D}(B, S \rightarrow j)=D_{\bar{\alpha}}(B, S \rightarrow j) \\
\text { where } \bar{\alpha}_{i}^{S \rightarrow j}=1 \text { for each } i \in I_{S \rightarrow j},
\end{gathered}
$$

and the positive equidistributed influence index of coalition $S$ on player $j$ is given by

$$
\begin{gathered}
D^{*}(B, S \rightarrow j)=D_{\alpha^{*}}(B, S \rightarrow j) \\
\text { where } \alpha_{i}^{* S \rightarrow j}=\frac{1}{\sum_{a \in A \backslash\left(A^{0} \cup\left\{i_{j}\right\}\right)}\left(2^{n^{a}(S, j, i)}-1\right)} \text { for each } i \in I_{S \rightarrow j} .
\end{gathered}
$$

Remark 2 Given $B \in \mathcal{B}, S \subseteq N, j \in N \backslash S$, if for each $i \in I_{S \rightarrow j}^{\text {pos }}(B)$, the decision of player $j$ 'lies' between his inclination and the inclination of coalition $S$, i.e., if for each $i \in I_{S \rightarrow j}^{p o s}(B)$

$$
\left|i_{j}-i_{S}\right|=\left|(B i)_{j}-i_{j}\right|+\left|(B i)_{j}-i_{S}\right|
$$

then the positive weighted influence index of coalition $S$ on player $j$ is equal to

$$
D_{\alpha}(B, S \rightarrow j):=\frac{\sum_{i \in I_{S \rightarrow j}^{\text {pos }}(B)}\left|(B i)_{j}-i_{j}\right| \alpha_{i}^{S \rightarrow j}}{\sum_{i \in I_{S \rightarrow j}}\left|i_{j}-i_{S}\right| \alpha_{i}^{S \rightarrow j}} .
$$

Remark 3 Note that if for a certain $i \in I_{S}, i_{j} \neq i_{S}$, but $\left|(B i)_{j}-i_{S}\right|>\left|i_{j}-i_{S}\right|$, the difference $\left|i_{j}-i_{S}\right|$ for such an $i$ is included in the denominator of equation (12), but we write just 0 in the nominator of equation $(12)$, since $i \notin I_{S \rightarrow j}^{\text {pos }}(B)$.

Remark 4 Note that we do not distinguish between two situations in which the decisions of the influenced player are different, but their distances from the inclination of the coalition are the same. Let us consider the situation mentioned above, in which the decision of the influenced player 'lies' between his inclination and the inclination of the coalition. Next, let us imagine that the influenced player 'puts' his decision on the 'other side' of the inclination of the coalition, farther from his own inclination, but the distance between the decision and the inclination of the coalition is the same as before. If this holds for each $i \in I_{S \rightarrow j}^{p o s}(B)$, we get the same positive weighted influence index of the coalition on the player.

Fact 1 Given $B \in \mathcal{B}, S \subseteq N, j \in N \backslash S$, if $\bar{D}(B, S \rightarrow j)=1$, then $\bar{d}(B, S \rightarrow j)=1$.

Proof: Suppose $\bar{D}(B, S \rightarrow j)=1$. This means that

$$
\sum_{i \in I_{S \rightarrow j}^{\text {pos }}(B)}\left[\left|i_{j}-i_{S}\right|-\left|(B i)_{j}-i_{S}\right|\right]=\sum_{i \in I_{S \rightarrow j}}\left|i_{j}-i_{S}\right|
$$

For each $i \in I_{S \rightarrow j}$, either $i \in I_{S \rightarrow j}^{p o s}(B)$, or $\left|i_{j}-i_{S}\right|<\left|(B i)_{j}-i_{S}\right|$. By virtue of (18), if $i \in I_{S \rightarrow j}^{p o s}(B)$, then $\left|(B i)_{j}-i_{S}\right|=0$, and therefore $(B i)_{j}=i_{S} \neq i_{j}$. If $\left|i_{j}-i_{S}\right|<\left|(B i)_{j}-i_{S}\right|$, then from (18) $\left|i_{j}-i_{S}\right|=0$, and therefore $i_{j}=i_{S}$ and $\left|(B i)_{j}-i_{S}\right|>0$. This means that $(B i)_{j} \neq i_{S}=i_{j}$. Hence, for each $i \in I_{S \rightarrow j},(B i)_{j} \neq i_{j}$, which gives $I_{S \rightarrow j}^{*}(B)=I_{S \rightarrow j}$. 


\subsection{The negative influence indices}

The concept of the influence adopted in this subsection is related to the opposite influence of a coalition on a player in the yes-no model defined in [3]. Under the opposite influence in the yes-no model, the inclinations of the coalition and the player coincide, but the decision of the player differs from his inclination. When measuring a negative influence of a coalition on a player in the model with an ordered set of possible actions, we look for the inclination vectors, in which the inclination of the player is different from the action(s) placed farthest from the inclination of the coalition. If the decision of the player is farther from the inclination of the coalition than his inclination was, we say that such an inclination vector is the inclination vector of (observed) negative influence of the coalition on the player. Of course, the goal of the influencing coalition in the case of the negative influence is different from the goal of such a coalition in the case of the positive influence. While in the case of the positive influence, the coalition tries to 'attract' the player's decision as close to its inclination as possible, when considering the negative influence, the goal of the coalition is to push the decision of the player as far from its inclination as possible.

Below, we formalize the concepts of the negative influence. For each $a \in A \backslash A^{0}$, we define

$$
M(a):=\left\{\widetilde{a} \in A\left|\widetilde{a}=\arg \max _{a^{\prime} \in A}\right| a-a^{\prime} \mid\right\} .
$$

Of course, $|M(a)| \in\{1,2\}$. In particular, for each $S \subseteq N$ and $i \in I_{S}$, the coalition $S$ has its inclination $i_{S}$, and then $M\left(i_{S}\right)$ is the set of all possible actions which are the farthest actions from the inclination $i_{S}$, and $M\left(i_{S}\right)$ may have either 1 or 2 elements.

For each $S \subseteq N$ and $j \in N \backslash S$, the set of all inclination vectors of potential negative influence of $S$ on $j$ is defined as

$$
\widetilde{I}_{S \rightarrow j}:=\left\{i \in I_{S} \mid i_{j} \notin M\left(i_{S}\right)\right\} .
$$

For each $S \subseteq N, j \in N \backslash S, B \in \mathcal{B}$, and $i \in \widetilde{I}_{S \rightarrow j}$

$$
\begin{gathered}
\widetilde{i}_{S}^{i_{j}}:=\arg \min _{\widetilde{i}_{S} \in M\left(i_{S}\right)}\left|i_{j}-\widetilde{i}_{S}\right| \\
\widetilde{i}_{S}^{(B i)_{j}}:=\arg \min _{\widetilde{i}_{S} \in M\left(i_{S}\right)}\left|(B i)_{j}-\widetilde{i}_{S}\right| .
\end{gathered}
$$

Of course, if $\left|M\left(i_{S}\right)\right|=1$, then $\widetilde{i}_{S}^{i_{j}}=\widetilde{i}_{S}^{(B i)_{j}}$.

The set of all inclination vectors of observed negative influence of $S$ on $j$ is defined as

$$
\widetilde{I}_{S \rightarrow j}^{n e g}(B):=\left\{i \in \widetilde{I}_{S \rightarrow j}|| i_{j}-\widetilde{i}_{S}^{i_{j}}|>|(B i)_{j}-\widetilde{i}_{S}^{(B i)_{j}} \mid\right\}
$$

In a similar way as before, for each $S \subseteq N, j \in N \backslash S$ and $i \in \widetilde{I}_{S \rightarrow j}$, we introduce a weight $\alpha_{i}^{S \rightarrow j} \in[0,1]$ of influence of coalition $S$ on $j \in N \backslash S$ under the inclination vector $i \in \widetilde{I}_{S \rightarrow j}$, where $\alpha_{i}^{S \rightarrow j}$ depends now on numbers $n^{a}(S, j, i)$, for $a \in A \backslash A^{0}$. 
Definition 3 Given $B \in \mathcal{B}$, for each $S \subseteq N, j \in N \backslash S$, the negative weighted influence index of coalition $S$ on player $j$ is defined as

$$
D_{\alpha}^{n e g}(B, S \rightarrow j):=\frac{\sum_{i \in \widetilde{I}_{S \rightarrow j}^{n e g}(B)}\left[\left|i_{j}-\widetilde{i}_{S}^{i j}\right|-\left|(B i)_{j}-\widetilde{i}_{S}^{(B i)_{j}}\right|\right] \alpha_{i}^{S \rightarrow j}}{\sum_{i \in \widetilde{I}_{S \rightarrow j}}\left|i_{j}-\widetilde{i}_{S}^{i j}\right| \alpha_{i}^{S \rightarrow j}} \in[0,1] .
$$

The negative possibility influence index of coalition $S$ on player $j$ is given by

$$
\bar{D}^{n e g}(B, S \rightarrow j):=\frac{\sum_{i \in \widetilde{I}_{S \rightarrow j}^{n e g}(B)}\left[\left|i_{j}-\widetilde{i}_{S}^{i_{j}}\right|-\left|(B i)_{j}-\widetilde{i}_{S}^{(B i)_{j}}\right|\right]}{\sum_{i \in \widetilde{I}_{S \rightarrow j}}\left|i_{j}-\widetilde{i}_{S}^{i_{j}}\right|} \in[0,1] .
$$

\subsection{The Examples}

The aim of this section is to show a broad applicability of the generalized framework and the influence indices that are studied in the paper. Several examples are mentioned below, while for two of them we calculate all concepts introduced in the paper.

Example 1 One of the natural applications of the model with an ordered set of actions is an application to politics, in particular to elections. An ideological line indicating a political orientation, from the Left Wing via the Centre to the Right Wing candidates or parties, has its natural interpretation here. Let us consider a French family before the first round of French presidential elections in Spring 2007. We assume that the family members consider to vote for one of three candidates, i.e., using the terminology of our model, each player has an inclination to choose one of the three actions: to vote for Segolène Royal (Action -1), to vote for Francois Bayrou (Action 0), or to vote for Nicolas Sarkozy $($ Action +1$)$. Consequently, neither abstaining nor voting for a candidate different from the three major ones is included in the set of possible actions of the family members. This situation is then modeled as a three-action model, but with the empty set of neutral actions, $A=\{-1,0,+1\}, A^{0}=\emptyset$. One can imagine here many kinds of an influence: the influence of the parents on the children, the influence of the husband on the wife, etc. Of course, the analysis would end at the stage of calculating the influence indices, since the group decision is determined on the country level, not on a family level.

Example 2 As mentioned before, a particular case of our generalized framework is a yes-no-abstention model, in which each player has an inclination either to say 'yes' or 'no', or to abstain. Let us check how the concepts and formulas introduced above look like for this three-action example. We have $A=\{-1,0,+1\}=C, A^{0}=\{0\}$,

$$
\begin{gathered}
I_{S}=\left\{i \in I \mid \forall k, j \in S\left[i_{k}=i_{j} \wedge i_{k} \neq 0\right\}\right. \\
I_{S \rightarrow j}=\left\{i \in I_{S} \mid i_{j} \neq i_{S}\right\} \\
I_{S \rightarrow j}^{*}(B)=\left\{i \in I_{S \rightarrow j} \mid(B i)_{j} \neq i_{j}\right\}
\end{gathered}
$$

$\alpha_{i}^{S \rightarrow j}$ depends on the number $n^{i_{S}}(S, j, i)$, where

$$
n^{i_{S}}(S, j, i)=\left|\left\{m \in N \backslash j \mid i_{m}=i_{S}\right\}\right|
$$




$$
\begin{gathered}
d_{\alpha}(B, S \rightarrow j)=\frac{\sum_{i \in I_{S \rightarrow j}^{*}(B)} \alpha_{i}^{S \rightarrow j}}{\sum_{i \in I_{S \rightarrow j}} \alpha_{i}^{S \rightarrow j}} \\
\bar{d}(B, S \rightarrow j)= \\
\frac{\left|I_{S \rightarrow j}^{-0-}(B)\right|+\left|I_{S \rightarrow j}^{-+0}(B)\right|+\left|I_{S \rightarrow j}^{+0+}(B)\right|+\left|I_{S \rightarrow j}^{+-0}(B)\right|+\left|I_{S \rightarrow j}^{++}(B)\right|+\left|I_{S \rightarrow j}^{+-+}(B)\right|+\left|I_{S \rightarrow j}^{-0+}(B)\right|+\left|I_{S \rightarrow j}^{+0-}(B)\right|}{4 \cdot 3^{n-s-1}}
\end{gathered}
$$

where for $a \in\{-1,+1\}, b, c \in\{-1,0,+1\}$

$$
\begin{gathered}
I_{S \rightarrow j}^{a b c}(B):=\left\{i \in I_{S} \mid i_{S}=a \wedge i_{j}=b \wedge(B i)_{j}=c\right\} \\
d^{*}(B, S \rightarrow j)=d_{\alpha^{*}}(B, S \rightarrow j), \quad \text { where } \quad \alpha_{i}^{* S \rightarrow j}=\frac{1}{2^{n^{i} S(S, j, i)}-1} \\
\bar{D}(B, S \rightarrow j)= \\
\frac{\left|I_{S \rightarrow j}^{-0-}(B)\right|+\left|I_{S \rightarrow j}^{-+0}(B)\right|+\left|I_{S \rightarrow j}^{+0+}(B)\right|+\left|I_{S \rightarrow j}^{+-0}(B)\right|+2\left(\left|I_{S \rightarrow j}^{-+-}(B)\right|+\left|I_{S \rightarrow j}^{+-+}(B)\right|\right)}{2 \cdot 3^{n-s}}
\end{gathered}
$$

For $a \in\{-1,+1\}, M(a)=\{-a\}$

$$
\begin{gathered}
\widetilde{I}_{S \rightarrow j}=\left\{i \in I_{S}|| i_{j}-i_{S} \mid \in\{0,1\}\right\} \\
\widetilde{i}_{S}^{i_{j}}=\widetilde{i}_{S}^{(B i)_{j}}=-i_{S} \\
\widetilde{I}_{S \rightarrow j}^{n e g}(B)=\left\{i \in I_{S}|| i_{j}+i_{S}|>|(B i)_{j}+i_{S} \mid\right\} \\
\bar{D}^{n e g}(B, S \rightarrow j)= \\
\frac{\left|I_{S \rightarrow j}^{-0+}(B)\right|+\left|I_{S \rightarrow j}^{--0}(B)\right|+\left|I_{S \rightarrow j}^{+0-}(B)\right|+\left|I_{S \rightarrow j}^{++0}(B)\right|+2\left(\left|I_{S \rightarrow j}^{--+}(B)\right|+\left|I_{S \rightarrow j}^{++-}(B)\right|\right)}{2 \cdot 3^{n-s}}
\end{gathered}
$$

Example 3 Let us consider the following example entitled 'Old or New'. A three-member committee is to choose a new researcher for a research lab. There is one position available, and two applicants. The committee consists of a director of the lab (player 1), and two professors who chair two different sections. The candidates have different research profiles: one candidate does research which coincides with the expertise of the lab, while research program of another candidate is far from topics the lab works on. The committee members use a criterion 'publication rate in a new research field'. Consequently, each committee member must choose one of the following three actions that we place on the criterion line:

- to choose candidate Mr. Old (Action -1) - this candidate would bring new publications in some fields of the expertise of the lab, but clearly would not bring to the lab any publications in a new research field;

- to choose nobody (to abstain - Action 0) - the publications of the lab would remain the same; or

- to choose candidate Mr. New (Action +2) - this candidate would bring to the lab many publications in a new research field. 
Each committee member has an inclination to choose one of the actions, but due to some influence of the others, he can vote differently than he preliminary felt like. It is assumed that the director of the lab always votes according to his preliminary inclination. If the director and another professor both want to choose the same candidate, Mr. Old or Mr. New, the remaining colleague will follow their inclination. The boss influences on his own a colleague with the inclination to abstain, but an abstaining committee member (also the director) has no ability to influence the others.

Using the notation introduced in the paper, we have the committee (the set of players) $N=\{1,2,3\}$, the set of actions $A=\{-1,0,+2\}=C$, and the abstention as the neutral action $A^{0}=\{0\}$. Moreover, there are 27 possible inclination vectors, $|I|=27$. Table 1 shows the inclination vectors and the decision vectors.

Table 1. The inclination and decision vectors

\begin{tabular}{c|c||c|c||c|c}
$i \in I$ & $B i$ & $i \in I$ & $B i$ & $i \in I$ & $B i$ \\
\hline \hline$(-1,-1,-1)$ & $(-1,-1,-1)$ & $(0,0,0)$ & $(0,0,0)$ & $(2,2,2)$ & $(2,2,2)$ \\
\hline$(-1,-1,0)$ & $(-1,-1,-1)$ & $(0,0,-1)$ & $(0,0,-1)$ & $(2,2,-1)$ & $(2,2,2)$ \\
\hline$(-1,0,-1)$ & $(-1,-1,-1)$ & $(0,-1,0)$ & $(0,-1,0)$ & $(2,-1,2)$ & $(2,2,2)$ \\
\hline$(0,-1,-1)$ & $(0,-1,-1)$ & $(-1,0,0)$ & $(-1,-1,-1)$ & $(-1,2,2)$ & $(-1,2,2)$ \\
\hline$(-1,-1,2)$ & $(-1,-1,-1)$ & $(0,0,2)$ & $(0,0,2)$ & $(2,2,0)$ & $(2,2,2)$ \\
\hline$(-1,2,-1)$ & $(-1,-1,-1)$ & $(0,2,0)$ & $(0,2,0)$ & $(2,0,2)$ & $(2,2,2)$ \\
\hline$(2,-1,-1)$ & $(2,-1,-1)$ & $(2,0,0)$ & $(2,2,2)$ & $(0,2,2)$ & $(0,2,2)$ \\
\hline$(-1,0,2)$ & $(-1,-1,2)$ & $(0,-1,2)$ & $(0,-1,2)$ & $(2,-1,0)$ & $(2,-1,2)$ \\
\hline$(-1,2,0)$ & $(-1,2,-1)$ & $(0,2,-1)$ & $(0,2,-1)$ & $(2,0,-1)$ & $(2,2,-1)$
\end{tabular}

Note that for $S \subseteq\{2,3\}, D_{\alpha}(B, S \rightarrow 1)=0$, because $(B i)_{1}=i_{1}$ for each $i \in I$. The positive and general possibility influence indices are as follows:

$$
\begin{gathered}
\bar{D}(B, 1 \rightarrow 2)=\bar{D}(B, 1 \rightarrow 3)=\frac{5}{9}, \quad \bar{d}(B, 1 \rightarrow 2)=\bar{d}(B, 1 \rightarrow 3)=\frac{2}{3} \\
\bar{D}(B, 2 \rightarrow 3)=\bar{D}(B, 3 \rightarrow 2)=\bar{d}(B, 2 \rightarrow 3)=\bar{d}(B, 3 \rightarrow 2)=\frac{1}{3} \\
\bar{D}(B, 12 \rightarrow 3)=\bar{D}(B, 13 \rightarrow 2)=\bar{d}(B, 12 \rightarrow 3)=\bar{d}(B, 13 \rightarrow 2)=1 .
\end{gathered}
$$

When considering, for instance, the influence of player 1 on player 2 , we verify 12 inclination vectors in which player 1's inclination is both different from 0 and different from player 2's inclination. In the case of 8 out of these 12 inclination vectors, player 2 votes differently than his inclination was. Note however that the influence has different strength. For instance, when we look at the inclination vectors $(-1,0,-1)$ and $(-1,2,-1)$, in both cases the decision vector is equal to $(-1,-1,-1)$. Nevertheless, in the first case, player 2 has moved only from 0 to -1 , while in the second case, he changed from 2 to -1 .

There are two cases, related to the calculations of the influence index of player 2 on player 3 , which illustrate Remark 3 . Let us look at the inclination vectors $(-1,2,0)$ and $(2,-1,0)$. Since the inclinations of player 2 and player 3 are different, we include the differences $|2-0|=2$ and $|-1-0|=1$ in the denominator of $\bar{D}(B, 2 \rightarrow 3)$, and just 1 and 1 in the denominator of $\bar{d}(B, 2 \rightarrow 3)$. Nevertheless, since player 3 moved his 
decision farther from the inclination of player 2, these cases are not seen as a positive influence of player 2 on player 3 , and consequently 0 and 0 are written in the nominators of $\bar{D}(B, 2 \rightarrow 3)$ and $\bar{d}(B, 2 \rightarrow 3)$.

We calculate also the negative possibility influence indices which, in most cases except two indices, are equal to 0 . The positive values of these indices are equal to:

$$
\bar{D}^{\text {neg }}(B, 2 \rightarrow 3)=\bar{D}^{\text {neg }}(B, 3 \rightarrow 2)=\frac{(1-0)+(2-0)}{27}=\frac{1}{9} .
$$

For instance, the inclination vector $(-1,2,0)$ leads to the decision vector $(-1,2,-1)$, which counts for the negative influence of player 2 on player 3. Both in the denominator and the nominator of $\bar{D}^{\text {neg }}(B, 2 \rightarrow 3)$ we write 1 : the farthest action of player 2 with the inclination 2 is -1 , which is of a distance equal to 1 from the neutral inclination of player 3 , and player 3 decides for the action -1 . Moreover, the inclination vector $(2,-1,0)$ leads to the decision vector $(2,-1,2)$, and we write 2 both in the denominator and the nominator of $\bar{D}^{\text {neg }}(B, 2 \rightarrow 3)$. Nevertheless, we should be aware that although the negative influence index of player 2 on player 3 has a positive value, it is rather due to the positive influence of player 1 on player 3 . As it was described before, player 1 has the ability to make an abstaining player decide according to the inclination of player 1. In both inclination vectors $(-1,2,0)$ and $(2,-1,0)$, the inclinations of players 1 and 2 are as far from each other as possible. In a similar way we can analyze the case of $\bar{D}^{n e g}(B, 3 \rightarrow 2)$.

Example 4 The same committee of the research lab might also be deciding about a new budget to invite speakers for external seminars. The actions are placed on the line 'changing the seminar budget':

- Action -1 - to decrease the present seminar budget by 1000 euro;

- Action 0 - to keep the seminar budget unchanged;

- Action +2 - to increase the present seminar budget by 2000 euro.

Contrary to the previous example, one could imagine an abstaining coalition to have an influence on the other(s), i.e., we have here $A=\{-1,0,+2\}=C$, but $A^{0}=\emptyset$. Given Table 1, the influence indices will be, of course, different from the ones calculated in Example 3, since we also take into account now all inclination vectors with Action 0 as the inclination of a coalition.

\section{Following a coalition}

In this section, we focus on the positive influence. One of the concepts related to the positive influence is the concept of a follower of a given coalition, that is, a voter who always follows the inclination of the coalition in question. We assume that $C=A$.

Definition 4 Let $\emptyset \neq S \subseteq N$ and $B \in \mathcal{B}$. The set of followers of $S$ under $B$ is defined as

$$
F_{B}(S):=\left\{j \in N \mid \forall i \in I_{S}\left[(B i)_{j}=i_{S}\right]\right\} .
$$

For each $a \in A \backslash A^{0}$, the set of conditional a-followers of $S$ under $B$ is defined as

$$
F_{B}\left(S \mid i_{S}=a\right):=\left\{j \in N \mid \forall i \in I_{S}\left[i_{S}=a \Rightarrow(B i)_{j}=a\right]\right\} .
$$


We have then

$$
\bigcap_{a \in A \backslash A^{0}} F_{B}\left(S \mid i_{S}=a\right)=F_{B}(S)
$$

Proposition 1 Let $B \in \mathcal{B}$. Then the following holds:

(i) Whenever $S \cap T=\emptyset, F_{B}(S) \cap F_{B}(T)=\emptyset$.

(ii) $F_{B}$ is an isotone function $\left(S \subseteq S^{\prime}\right.$ implies $\left.F_{B}(S) \subseteq F_{B}\left(S^{\prime}\right)\right)$.

Consequently, if $F_{B}(N)=\emptyset$, then $F_{B} \equiv \emptyset$.

(iii) For each $j \in F_{B}(S) \backslash S, D_{\alpha}(B, S \rightarrow j)=1$, and $D_{\alpha}^{\text {neg }}(B, S \rightarrow j)=0$.

Proof: (i) Since $S \cap T=\emptyset, I_{S} \cap I_{T}$ strictly includes $I_{S \cup T}$. Then there exists $i \in I_{S} \cap I_{T}$ such that $i_{S} \neq i_{T}$. Hence, if $j \in F_{B}(S) \cap F_{B}(T)$, the equality $(B i)_{j}=i_{S}=i_{T}$ cannot hold for this $i$.

(ii) Take $S \subseteq S^{\prime}$ and $j \in F_{B}(S)$. $i \in I_{S^{\prime}}$ implies $i \in I_{S}$ by antitonicity, hence $(B i)_{j}=i_{S}$ by hypothesis, and since $i_{S}=i_{S^{\prime}}, j \in F_{B}\left(S^{\prime}\right)$.

(iii) Let $B \in \mathcal{B}, S \subset N, F_{B}(S) \neq \emptyset$, and $j \in F_{B}(S) \backslash S$. Hence, for each $i \in I_{S},(B i)_{j}=i_{S}$, and therefore $D_{\alpha}(B, S \rightarrow j)=1$, and $D_{\alpha}^{\text {neg }}(B, S \rightarrow j)=0$.

Assume $F_{B}$ is not identically the empty set. Then the kernel of $B$ is the following collection of sets:

$$
\mathcal{K}(B):=\left\{S \in 2^{N} \mid F_{B}(S) \neq \emptyset \text {, and } S^{\prime} \subset S \Rightarrow F_{B}\left(S^{\prime}\right)=\emptyset\right\} .
$$

The kernel is well defined due to isotonicity. It is the set of "true" influential coalitions.

Definition 5 Let $S, T$ be two disjoint non empty subsets of $N . B$ is said to be a purely influential function of $S$ upon $T$ if it satisfies for all $i \in I_{S}$ :

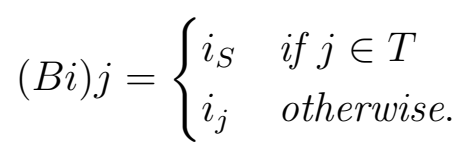

The set of such functions is denoted $\mathcal{B}_{S \rightarrow T}$.

Note that these functions are arbitrary on $I \backslash I_{S}$. What is the cardinality of $\mathcal{B}_{S \rightarrow T}$ ? We have

$$
\left|\mathcal{B}_{S \rightarrow T}\right|=|A|^{n\left(|A|^{s}-|A|+\left|A^{0}\right|\right)|A|^{n-s}}
$$

Proposition 2 Let $S, T$ be two disjoint non empty subsets of $N$. Then the following holds:

(i) For all $B \in \mathcal{B}_{S \rightarrow T}, F_{B}(S)=S \cup T$.

(ii) For each $B \in \mathcal{B}_{S \rightarrow T}$ and $j \in N \backslash S, D_{\alpha}^{\text {neg }}(B, S \rightarrow j)=0$, and

$$
D_{\alpha}(B, S \rightarrow j)= \begin{cases}1 & \text { if } j \in T \\ 0 & \text { if } j \in N \backslash(S \cup T)\end{cases}
$$


Proof: (i) Take $t \in S \cup T$. If $t \in T$, then for any $i \in I_{S},(B i)_{t}=i_{S}$. If $t \in S$, then for any $i \in I_{S},(B i)_{t}=i_{t}=i_{S}$. Hence $t \in F_{B}(S)$. On the other hand, take $t \in F_{B}(S)$. Then for any $i \in I_{S},(B i)_{t}=i_{S}$, and hence $t \in S \cup T$.

(ii) Let $B \in \mathcal{B}_{S \rightarrow T}$. Then for each $i \in I_{S},(B i)_{j}=i_{S}$ for $j \in T$, and $(B i)_{j}=i_{j}$ for $j \notin T$. Since $I_{S \rightarrow j} \subset I_{S}$, we have for each $i \in I_{S \rightarrow j},(B i)_{j}=i_{S}$ for $j \in T$, and $(B i)_{j}=i_{j}$ for $j \notin T$. Hence, if $j \in T$, then $D_{\alpha}(B, S \rightarrow j)=1$, and if $j \in N \backslash(S \cup T)$, then $D_{\alpha}(B, S \rightarrow j)=0$. Moreover, for each $j \in N \backslash S, D_{\alpha}^{\text {neg }}(B, S \rightarrow j)=0$.

Example 5 The sets of followers for Example 3 presented in Section 2.4 are the following:

$$
\begin{gathered}
F_{B}(\emptyset)=F_{B}(2)=F_{B}(3)=\emptyset, \quad F_{B}(1)=\{1\} \\
F_{B}(23)=\{2,3\}, \quad F_{B}(12)=F_{B}(13)=F_{B}(123)=\{1,2,3\}
\end{gathered}
$$

The sets of $a$-conditional followers are here the same as the sets of followers, i.e., for $a \in\{-1,2\}$, and $S \subset\{1,2,3\}, F_{B}\left(S \mid i_{S}=a\right)=F_{B}(S)$.

The kernel $\mathcal{K}(B)=\{\{1\},\{2,3\}\}$. Moreover, $B \in \mathcal{B}_{12 \rightarrow 3} \cap \mathcal{B}_{13 \rightarrow 2}$.

\section{The influence functions}

Next, we define several influence functions $B \in \mathcal{B}$ and investigate their properties. In particular, for each influence function analyzed, we determine the set of followers and the values of the (positive and negative) weighted influence indices.

Some simple examples of influence functions are:

(i) The Majority function. Let $n \geq t>\left\lfloor\frac{n}{2}\right\rfloor$, and introduce for any $i \in I$ and $a \in A \backslash A^{0}$, the set

$$
i^{a}:=\left\{k \in N \mid i_{k}=a\right\} .
$$

The majority influence function $\mathrm{Maj}^{[t]} \in \mathcal{B}$ is defined by

$$
\left(\operatorname{Maj}^{[t]} i\right)_{j}:=\left\{\begin{array}{ll}
a, & \text { if } \exists a \in A \backslash A^{0}\left[\left|i^{a}\right| \geq t\right] \\
i_{j}, & \text { otherwise }
\end{array}, \quad \forall i \in I, \quad \forall j \in N .\right.
$$

If a majority of players have an inclination $a$, then all players decide for $a$. If not, each player decides according to his own inclination.

(ii) The Guru function. Let $\widetilde{k} \in N$ be a particular player called the guru. The guru influence function Gur $^{[\widetilde{k}]} \in \mathcal{B}$ is defined by

$$
\left(\operatorname{Gur}^{[\widetilde{k}]} i\right)_{j}=i_{\widetilde{k}}, \quad \forall i \in I, \quad \forall j \in N
$$

When a guru exists, every player follows always the guru.

(iii) The identity function $\mathrm{Id} \in \mathcal{B}$ is defined by

$$
\operatorname{ld} i=i, \quad \forall i \in I \text {. }
$$


(iv) The mass psychology effect. Let $t \in(0, n]$ and $a \in A \backslash A^{0}$. Functions $B \in \mathcal{B}$ satisfying for each $i \in I$

$$
\text { if }\left|i^{a}\right| \geq t, \text { then }(B i)^{a} \supseteq i^{a}
$$

are called mass psychology influence functions. If there is a sufficiently high number of players with inclination $a$, no of these players will decide differently than $a$. We denote by $\mathcal{B}^{[a, t]}$ the set of such influence functions.

We list some basic properties of the influence functions mentioned.

Proposition 3 Let $n \geq t>\left\lfloor\frac{n}{2}\right\rfloor$ and consider the majority function Maj ${ }^{[t]}$. Then the following holds:

(i) For each $\emptyset \neq S \subseteq N$ such that $s \geq t$, and for each $j \in N \backslash S$, $D_{\alpha}\left(\mathrm{Maj}^{[t]}, S \rightarrow j\right)=1$, and $D_{\alpha}^{\text {neg }}\left(\mathrm{Maj}^{[t]}, S \rightarrow j\right)=0$

(ii) For each $S \subseteq N$,

$$
F_{\mathrm{Maj}[t]}(S)= \begin{cases}N, & \text { if } s \geq t \\ S, & \text { if } n-t<s<t \\ \emptyset, & \text { if } s \leq n-t .\end{cases}
$$

(iii) The kernel is $\mathcal{K}\left(\mathrm{Maj}^{[t]}\right)=\{S \subseteq N|| S \mid=n-t+1\}$.

Proof: (i) Let $\emptyset \neq S \subseteq N$ be such that $s \geq t$, and $j \in N \backslash S$. For each $i \in I_{S}$, there is $a \in A \backslash A^{0}$ such that $i_{S}=a$, and therefore $\left|i^{a}\right| \geq s \geq t$. Hence, $\left(\mathrm{Maj}^{\left[{ }^{[t]}\right.} i\right)_{j}=a=i_{S}$ for each $i \in I_{S}$, which gives $D_{\alpha}\left(\mathrm{Maj}^{[t]}, S \rightarrow j\right)=1$, and $D_{\alpha}^{n e g}\left(\mathrm{Maj}^{[t]}, S \rightarrow j\right)=0$.

(ii) Let $S \subseteq N$ be such that $s \geq t$. Suppose that $F_{\mathrm{Maj}[t]}(S) \neq N$. Then there exists $j \in N$ such that $j \notin F_{\mathrm{Maj}^{[t]}}(S)$. Hence, there is $\widetilde{i} \in I_{S}$ such that $\left(\mathrm{Maj}{ }^{[t \tau} \widetilde{i}\right)_{j} \neq \widetilde{i}_{S}$. But $\widetilde{i}_{S}=a$ for certain $a \in A \backslash A^{0}$, and $\left|\widetilde{i^{a}}\right| \geq s \geq t$. Hence, $\left(\operatorname{Maj}^{[t]} \widetilde{i}\right)_{j}=a=\widetilde{i}_{S}$, a contradiction.

Let $S \subseteq N$ be such that $n-t<s<t$, and therefore $s<t$ and $n-s<t$. Hence, for each $i \in I_{S}$, either there exists $a \in A \backslash A^{0}$ such that $\left|i^{a}\right| \geq t$ and $i_{S}=a$, and hence $\left(\mathrm{Maj}^{[t]} i\right)_{j}=a=i_{S}$ for each $j \in N$, or $\left(\mathrm{Maj}^{[t]} i\right)_{j}=i_{j}$ for each $j \in N$.

Note that $S \subseteq F_{\mathrm{Maj}^{[t]}}(S)$, because if $j \in S$, then for each $i \in I_{S}$, either $\left(\mathrm{Maj}^{[t]} i\right)_{j}=i_{S}$ or $\left(\mathrm{Maj}^{[t]} i\right)_{j}=i_{j}=i_{S}$.

Suppose $F_{\mathrm{Maj}}[t](S) \nsubseteq S$. Hence, there is $k \notin S$ such that $k \in F_{\mathrm{Maj}^{[t]}}(S)$, and therefore for each $i \in I_{S},\left(\mathrm{Maj}^{[t]} i\right)_{k}=i_{S}$. Take $\tilde{i} \in I_{S}$ such that $\widetilde{i}_{S} \neq \widetilde{i}_{k}=\widetilde{i}_{j}$ for each $j \notin S$. Then we have $\left(\operatorname{Maj}^{[t]} \widetilde{i}_{k}=\widetilde{i}_{k} \neq \widetilde{i}_{S}\right.$, a contradiction.

Let $S \subseteq N$ be such that $s \leq n-t$. Suppose that $F_{\mathrm{Maj}^{[t]}}(S) \neq \emptyset$, and let $\tilde{j} \in F_{\mathrm{Maj}^{[t]}}(S)$. Hence, for each $i \in I_{S}$, $\left(\mathrm{Maj}^{[t]} i\right)_{\tilde{j}}=i_{S}$. Take $\widetilde{i} \in I_{S}$ such that $\widetilde{i}_{S} \neq \widetilde{i}_{k}=a$ for each $k \notin S$ and certain $a \in A \backslash A^{0}$. Hence, $\left|\widetilde{i^{a}}\right|=n-s \geq t$, and therefore for each $j \in N$, $\left(\mathrm{Maj}^{[t]} \widetilde{i}\right)_{j}=a \neq \widetilde{i}_{S}$, a contradiction.

(iii) By virtue of (36), we have the following. If $|S| \leq n-t$, then $F_{\mathrm{Maj}[t]}(S)=\emptyset$, and hence $S \notin \mathcal{K}\left(\mathrm{Maj}^{[t]}\right)$. If $|S|=n-t+1$, then $F_{\mathrm{Maj}[t]}(S)=S$, but for each $S^{\prime} \subset S,\left|S^{\prime}\right| \leq n-t$, and therefore $F_{\mathrm{Maj}}\left(S^{\prime}\right)=\emptyset$. Hence, $S \in \mathcal{K}\left(S^{\prime}{ }^{\left[{ }^{[}\right]}\right)$. If $|S|>n-t+1$, then $F_{\mathrm{Maj}^{[t]}}(S) \neq \emptyset$, and there exists $S^{\prime} \subset S$ such that $\left|S^{\prime}\right| \geq n-t+1$, which means that $F_{\text {Maj }[t]}\left(S^{\prime}\right) \neq \emptyset$. 
Hence, $S \notin \mathcal{K}\left(\mathrm{Maj}^{[t]}\right)$.

Proposition 4 Let $\widetilde{k} \in N$ and consider the guru influence function Gur ${ }^{[\widetilde{k}]}$. Then the following holds:

(i) For each $\emptyset \neq S \subseteq N$ such that $\widetilde{k} \in S$, and for each $j \in N \backslash(S \cup\{\widetilde{k}\})$, $D_{\alpha}\left(\operatorname{Gur}^{[\tilde{k}]}, S \rightarrow j\right)=1$, and $D_{\alpha}^{n e g}\left(\operatorname{Gur}^{[\tilde{k}]}, S \rightarrow j\right)=0$.

(ii) For each $S \subseteq N$,

$$
F_{\text {Gur }[\tilde{k}]}(S)= \begin{cases}N, & \text { if } \widetilde{k} \in S \\ \emptyset, & \text { if } \widetilde{k} \notin S .\end{cases}
$$

(iii) The kernel is $\mathcal{K}\left(\operatorname{Gur}^{[\tilde{k}]}\right)=\{\widetilde{k}\}$.

(iv) $\operatorname{Gur}^{[\widetilde{k}]}$ is the unique purely influential function of $\widetilde{k}$ upon $N \backslash \widetilde{k}$, i.e., $\mathcal{B}_{\widetilde{k} \rightarrow N \backslash \widetilde{k}}=\left\{\mathrm{Gur}^{[\widetilde{k}]}\right\}$.

Proof: (iv) Gur ${ }^{[\widetilde{k}]} \in \mathcal{B}_{\widetilde{k} \rightarrow N \backslash \widetilde{k}}$ comes immediately from (28) and (33). Now, $\mathcal{B}_{\widetilde{k} \rightarrow N \backslash \widetilde{k}}$ is reduced to a singleton since $I \backslash I_{\widetilde{k}}=\emptyset$.

(i) Let $\emptyset \neq S \subseteq N$ be such that $\widetilde{k} \in S$, and $j \in N \backslash S$. Hence, in particular for each $i \in I_{S},\left(\operatorname{Gur}^{[\widetilde{k}]} i\right)_{j}=i_{\widetilde{k}}=i_{S}$, which gives $D_{\alpha}\left(\operatorname{Gur}^{[\widetilde{k}]}, S \rightarrow j\right)=1$. Moreover, $\widetilde{I}_{S \rightarrow j}^{n e g}(B)=\emptyset$, ad therefore $D_{\alpha}^{n e g}\left(\right.$ Gur $\left.^{[\widetilde{k}]}, S \rightarrow j\right)=0$.

(ii) The first line can be deduced from the fact that $F_{\text {Gur }}^{[\widetilde{k}]}$ is an isotone function and from Prop. 2 (i) since Gur ${ }^{[\widetilde{k}]}$ is purely influential by (iv).

Let $S \subseteq N$ be such that $\widetilde{k} \notin S$. Suppose that $F_{\text {Gur }[\tilde{k}]}(S) \neq \emptyset$. Let $\widetilde{j} \in F_{\text {Gur }[\tilde{k}]}(S)$. Hence, for each $i \in I_{S},\left(\operatorname{Gur}^{[\widetilde{k}]} i\right)_{\tilde{j}}=i_{S}$. Take $\widetilde{i} \in I_{S}$ such that $\widetilde{i}_{S} \neq \widetilde{i}_{k}$. Hence, for each $j \in N$, $\left(\operatorname{Gur}^{[\widetilde{k}]} \tilde{i}\right)_{j}=\widetilde{i}_{\widetilde{k}} \neq \widetilde{i}_{S}$, and in particular, $\left(\operatorname{Gur}^{[\widetilde{k}} \vec{i}\right)_{\tilde{j}}=\widetilde{i}_{\widetilde{k}} \neq \widetilde{i}_{S}$, a contradiction.

(iii) clear from (37).

Proposition 5 Let us consider the identity function Id. Then the following holds:

(i) For each $\emptyset \neq S \subseteq N$ and $j \in N \backslash S, D_{\alpha}(\mathrm{Id}, S \rightarrow j)=D_{\alpha}^{\text {neg }}(\mathrm{Id}, S \rightarrow j)=0$.

(ii) For each $S \subseteq N, F_{\text {Id }}(S)=S$.

(iii) The kernel is $\mathcal{K}(\mathrm{Id})=\{\{k\}, k \in N\}$.

Proof: (i) For each $\emptyset \neq S \subseteq N$ and $j \in N \backslash S,(B i)_{j}=i_{j}$ for $i \in I$, and therefore $D_{\alpha}(\mathrm{Id}, S \rightarrow j)=0$, and $D_{\alpha}^{\text {neg }}(\mathrm{Id}, S \rightarrow j)=0$.

(ii) Note that $S \subseteq F_{\text {ld }}(S)$, because if $j \in S$, then in particular for each $i \in I_{S}$, $(\operatorname{Id} i)_{j}=$ $i_{j}=i_{S}$. Suppose $F_{\text {ld }}(S) \nsubseteq S$. Hence, there is $k \notin S$ such that $k \in F_{\text {ld }}(S)$, and therefore for each $i \in I_{S},(\operatorname{Id} i)_{k}=i_{S}$. Take $\widetilde{i} \in I_{S}$ such that $\widetilde{i}_{S} \neq \widetilde{i}_{k}$. Then we have $(\operatorname{Id} \widetilde{i})_{k}=\widetilde{i}_{S} \neq \widetilde{i}_{k}$, but I I $\widetilde{i}=\widetilde{i}$, a contradiction.

(iii) Clear from (ii). 
Proposition 6 Let $t \in(0, n]$ and $a \in A \backslash A^{0}$ be fixed, and consider any influence function $B$ in $\mathcal{B}^{[a, t]}$. Then the following holds:

(i) There exists $B \in \mathcal{B}^{[a, t]}$ such that for each $\emptyset \neq S \subseteq N$ and $j \in N \backslash S$,

$$
D_{\alpha}(\mathrm{Id}, S \rightarrow j)=D_{\alpha}^{n e g}(\mathrm{Id}, S \rightarrow j)=0 .
$$

(ii) For each $\emptyset \neq S \subseteq N$ such that $s>n-t, t>1$, and $j \in N \backslash S$, there exists $B \in \mathcal{B}^{[a, t]}$ such that

$$
D_{\alpha}(B, S \rightarrow j)=1
$$

(iii) For each $S \subseteq N$,

$$
F_{B}(S) \subseteq S \text { if } s \leq n-t .
$$

Moreover, there exists $B \in \mathcal{B}^{[a, t]}$ such that for each $S \subseteq N, F_{B}(S)=S$.

Proof: (i) Remark that Id $\in \mathcal{B}^{[a, t]}$ for any $a \in A \backslash A^{0}$ and any $t \in(0, n]$. By virtue of Proposition 5, (i), we get $D_{\alpha}(\mathrm{Id}, S \rightarrow j)=0$ and $D_{\alpha}^{\text {neg }}(\mathrm{Id}, S \rightarrow j)=0$ for each $\emptyset \neq S \subseteq N$ and $j \in N \backslash S$.

(ii) Take arbitrary $\emptyset \neq S \subseteq N$ such that $s>n-t, t>1$, and $j \notin S$. Define $B$ as follows:

$$
(B i)_{k}= \begin{cases}i_{S}, & \text { if } i \in I_{S \rightarrow j} \text { and } k=j \\ i_{k}, & \text { otherwise }\end{cases}
$$

Note that $B$ belongs to $\mathcal{B}^{[a, t]}$, because if $i \in I_{S \rightarrow j}^{a^{\prime}}$ for $a^{\prime} \neq a$, where

$$
I_{S \rightarrow j}^{a^{\prime}}=\left\{i \in I_{S \rightarrow j} \mid i_{S}=a^{\prime}\right\}
$$

then $\left|i^{a}\right|<t$, and if $i \in I \backslash I_{S \rightarrow j}^{a^{\prime}}$, then (35) is satisfied. We have $D_{\alpha}(B, S \rightarrow j)=1$.

(iii) Let $t \in(0, n], a \in A \backslash A^{0}$, and $s \leq n-t$. Suppose there is $B \in \mathcal{B}^{[a, t]}$ such that $F_{B}(S) \nsubseteq S$ for a certain $S \subseteq N$. This means that $F_{B}(S) \neq \emptyset$, since $\emptyset \subseteq S$ for each $S$. Hence, there is $k \notin S$ such that $k \in F_{B}(S)$. This means that for each $i \in I_{S},(B i)_{k}=i_{S}$. Take $\widetilde{i} \in I_{S}$ such that $\widetilde{i}_{S} \neq a, \widetilde{i}_{k}=a$, and $\left|\widetilde{i}^{a}\right| \geq t$. Such an $\widetilde{i}$ always exists, because $n-s \geq t$. We have $(\widetilde{B i})_{k}=\widetilde{i}_{S} \neq a$, and therefore $(\widetilde{i})^{a} \nsubseteq(\widetilde{B i})^{a}$. But since $\left|\widetilde{i^{a}}\right| \geq t$, we should have $\widetilde{(i})^{a} \subseteq(\widetilde{B i})^{a}$, a contradiction.

If we take $B=\mathrm{Id}$, then for each $S \subseteq N, F_{\mathrm{Id}}(S)=S$.

\section{On Success, Failure, and Decisiveness}

In this section we generalize the concepts of Success, Decisiveness, Luck, and Failure of a player (see [6] and [3]) for the multi-choice model, by introducing a parameter $\beta$, where

$$
\beta \leq \max _{a \in C, a^{\prime} \in G}\left|a-a^{\prime}\right|
$$

Consequently, we consider $\beta$-Success, $\beta$-Failure, $\beta$-Luck, and $\beta$-Decisiveness, where for instance $\beta$-Success of a player is defined as a probability that the player is $\beta$-successful, 
meaning that a 'distance' between his decision and the group decision is not greater than $\beta$. $\beta$-Failure of a player is a probability that the player is not $\beta$-successful, i.e., a 'distance' between his decision and the group decision is greater than $\beta$. A player is $\beta$-decisive if he is $\beta$-successful, and under an arbitrary change of his decision, a distance between his new decision and the (new) group decision is not greater than the distance between the preliminary decision and the resulting group decision. A player is $\beta$-lucky if he is $\beta$-successful, but not $\beta$-decisive.

Similar as in [3], given a probability distribution $p: I \rightarrow[0,1]$ over all inclination vectors, and $B \in \mathcal{B}$, we define $p_{B}=p \circ B^{-1}$ on $C^{n}$ (probability of the decision vectors). Furthermore, the group decision function $g d: C^{n} \rightarrow G$ assigns to each decision vector $b \in C^{n}$ the value $a \in G$ if the group decision is to choose $a$.

Moreover, for $b \in c^{n}, k \in N$, and $a \in A \backslash\left\{b_{k}\right\}$, we define $b^{k, a} \in C^{n}$ by

$$
b_{j}^{k, a}=\left\{\begin{array}{ll}
b_{j} & \text { if } j \neq k \\
a & \text { if } j=k
\end{array} .\right.
$$

Definition 6 Given $\beta, g d \in \mathcal{G}, p_{B}: C^{n} \rightarrow[0,1]$, we define for each $k \in N$

- $\beta$-Success of player $k$

$$
\mathrm{SUC}_{k}^{\beta}\left(g d, p_{B}\right):=\operatorname{Pr}(k \text { is } \beta \text {-successful })=\sum_{\left\{b \in C^{n} \| b_{k}-g d(b) \mid \leq \beta\right\}} p_{B}(b)
$$

- $\beta$-Failure of player $k$

$$
\operatorname{FAIL}_{k}^{\beta}\left(g d, p_{B}\right):=1-\operatorname{SUC}_{k}^{\beta}\left(g d, p_{B}\right)=\sum_{\left\{b \in C^{n}|| b_{k}-g d(b) \mid>\beta\right\}} p_{B}(b)
$$

- $\beta$-Decisiveness of player $k$

$$
\operatorname{DEC}_{k}^{\beta}\left(g d, p_{B}\right):=\operatorname{Pr}(k \text { is } \beta \text {-decisive })=\sum_{\left\{b \in C^{n}|\beta \geq| b_{k}-g d(b)|\geq| a-g d\left(b^{k, a}\right) \mid \forall a \neq b_{k}\right\}} p_{B}(b)
$$

- $\beta$-Luck of player $k$

$$
\operatorname{LUCK}_{k}^{\beta}\left(g d, p_{B}\right):=\operatorname{Pr}(k \text { is } \beta \text {-lucky })=\sum_{\left\{b \in C^{n}|\beta \geq| b_{k}-g d(b)|<| a-g d\left(b^{k, a}\right) \mid \text { for some } a \neq b_{k}\right\}} p_{B}(b)
$$

According to [1], the following relation between Success, Luck, and Decisiveness holds:

$$
\text { Success }=\text { Decisiveness }+ \text { Luck }
$$

and in our case, we have for each $\beta, k \in N, p_{B}$, and $g d \in \mathcal{G}$

$$
\operatorname{SUC}_{k}^{\beta}\left(g d, p_{B}\right)=\operatorname{DEC}_{k}^{\beta}\left(g d, p_{B}\right)+\operatorname{LUCK}_{k}^{\beta}\left(g d, p_{B}\right) .
$$

Note that $\beta$-Success and $\beta$-Failure are monotone with respect to $\beta$, i.e., for each $k \in N, p_{B}$, and $g d \in \mathcal{G}$

$$
\text { if } \beta>\widetilde{\beta} \text {, then } \mathrm{SUC}_{k}^{\beta}\left(g d, p_{B}\right) \geq \operatorname{SUC}_{k}^{\widetilde{\beta}}\left(g d, p_{B}\right) \text { and } \operatorname{FAIL}_{k}^{\beta}\left(g d, p_{B}\right) \leq \operatorname{FAIL}_{k}^{\widetilde{\beta}}\left(g d, p_{B}\right) \text {. }
$$

We have also for each $k \in N, p_{B}$, and $g d \in \mathcal{G}$

$$
\operatorname{SUCC}_{k}^{\max _{a \in C, a^{\prime} \in G}\left|a-a^{\prime}\right|}\left(g d, p_{B}\right)=1, \quad \operatorname{FAIL}_{k}^{\max _{a \in C, a^{\prime} \in G}\left|a-a^{\prime}\right|}\left(g d, p_{B}\right)=0 .
$$


Example 6 We come back to Example 3, and assume that all inclination vectors are equally probable, that is,

$$
p(i)=\frac{1}{27} \text { for each } i \in I .
$$

The group decision is defined for each $b \in C^{n}$ as:

$$
g d(b)= \begin{cases}a & \text { if }\left|\left\{k \in N \mid b_{k}=a\right\}\right| \geq 2 \\ b_{1} & \text { otherwise. }\end{cases}
$$

which means that if a majority of the committee members chooses the same action, this action becomes the group decision, and if all three members choose different actions, this is the director of the lab (player 1) who decides. By virtue of (50) and Table 1, we get Table 2 .

Table 2. The group decision

\begin{tabular}{c|c|c||c|c|c||c|c|c}
$b \in C^{n}$ & $p_{B}(b)$ & $g d(b)$ & $b \in C^{n}$ & $p_{B}(b)$ & $g d(b)$ & $b \in C^{n}$ & $p_{B}(b)$ & $g d(b)$ \\
\hline$(-1,-1,-1)$ & $6 / 27$ & -1 & $(0,0,0)$ & $1 / 27$ & 0 & $(2,2,2)$ & $6 / 27$ & 2 \\
\hline$(-1,-1,0)$ & 0 & -1 & $(0,0,-1)$ & $1 / 27$ & 0 & $(2,2,-1)$ & $1 / 27$ & 2 \\
\hline$(-1,0,-1)$ & 0 & -1 & $(0,-1,0)$ & $1 / 27$ & 0 & $(2,-1,2)$ & $1 / 27$ & 2 \\
\hline$(0,-1,-1)$ & $1 / 27$ & -1 & $(-1,0,0)$ & 0 & 0 & $(-1,2,2)$ & $1 / 27$ & 2 \\
\hline$(-1,-1,2)$ & $1 / 27$ & -1 & $(0,0,2)$ & $1 / 27$ & 0 & $(2,2,0)$ & 0 & 2 \\
\hline$(-1,2,-1)$ & $1 / 27$ & -1 & $(0,2,0)$ & $1 / 27$ & 0 & $(2,0,2)$ & 0 & 2 \\
\hline$(2,-1,-1)$ & $1 / 27$ & -1 & $(2,0,0)$ & 0 & 0 & $(0,2,2)$ & $1 / 27$ & 2 \\
\hline$(-1,0,2)$ & 0 & -1 & $(0,-1,2)$ & $1 / 27$ & 0 & $(2,-1,0)$ & 0 & 2 \\
\hline$(-1,2,0)$ & 0 & -1 & $(0,2,-1)$ & $1 / 27$ & 0 & $(2,0,-1)$ & 0 & 2
\end{tabular}

Success, Failure, Decisiveness and Luck for each player and $\beta \in\{0,1,2\}$ are presented in Table 3.

Table 3. Success, Failure, Decisiveness and Luck

\begin{tabular}{c||c|c|c|c|c|c|c|c|c|c|c|c|c|c}
$k$ & SUC $_{k}^{0}$ & FAIL $_{k}^{0}$ & DEC $_{k}^{0}$ & LUCK $_{k}^{0}$ & SUC $_{k}^{1}$ & FAIL $_{k}^{1}$ & DEC $_{k}^{1}$ & LUCK $_{k}^{1}$ & SUC $_{k}^{2}$ & FAIL $_{k}^{2}$ & DEC $_{k}^{2}$ & LUCK $_{k}^{2}$ \\
\hline \hline 1 & $23 / 27$ & $4 / 27$ & $10 / 27$ & $13 / 27$ & $24 / 27$ & $3 / 27$ & $10 / 27$ & $14 / 27$ & $25 / 27$ & $2 / 27$ & $10 / 27$ & $15 / 27$ \\
\hline 2 & $21 / 27$ & $6 / 27$ & 0 & $21 / 27$ & $23 / 27$ & $4 / 27$ & $1 / 27$ & $22 / 27$ & $25 / 27$ & $2 / 27$ & $2 / 27$ & $23 / 27$ \\
\hline 3 & $21 / 27$ & $6 / 27$ & 0 & $21 / 27$ & $23 / 27$ & $4 / 27$ & $1 / 27$ & $22 / 27$ & $25 / 27$ & $2 / 27$ & $2 / 27$ & $23 / 27$
\end{tabular}

\section{Conclusions}

The main improvement of this paper is to enlarge the set of possible actions in the original framework of influence in a social network, in which players have only two inclinations and two possible decisions. While in the original framework only a yes-no decision was considered, in the present paper we analyze a multi-choice game, where each player has a totally ordered set of possible actions. A player has an inclination to choose one of the actions, but due to the influence by the others, his decision may be different from his inclination. We define the generalized (weighted) influence indices, and distinguish 
several influence indices with particular weights. The positive influence and the negative influence are measured. To the best of our knowledge, the concept of the generalized influence indices has not been proposed before. Hence, it is a new and interesting concept from a theoretical point of view, but also its applicability is worth remarking. Combining multi-choice problems with influence aspects creates a challenging framework to study.

There are several possibilities for conducting future research on this topic. One of them is to introduce dynamic aspects into this framework, and to analyze the iteration of the influence. Another interesting generalization would be to assume that the influence function $B$ is a probabilistic function. Furthermore, we would like to find an axiomatic characterization of the influence indices.

\section{References}

1. B. Barry. Is it better to be powerful or lucky?, Part I and Part II. Political Studies, 28:183-194, 338-352, 1980.

2. D. Felsenthal and M. Machover. Ternary voting games. International Journal of Game Theory, 26:335-351, 1997.

3. M. Grabisch and A. Rusinowska. The influence indices. GATE Working Paper, 2007-5, GATE, Ecully, France, 2007.

4. C. Hoede and R. Bakker. A theory of decisional power. Journal of Mathematical Sociology, 8:309-322, 1982.

5. C. R. Hsiao and T. E. S. Raghavan. Shapley value for multichoice cooperative games. Games and Economic Behavior, 5:240-256, 1993.

6. A. Laruelle and F. Valenciano. Assessing success and decisiveness in voting situations. Social Choice and Welfare, 24:171-197, 2005.

7. A. Rusinowska. On the not-preference-based Hoede-Bakker index. In L. Petrosjan and V. Mazalov, editors, Game Theory and Applications, volume 13, New York, 2008. Nova Science Publishers, Inc.

8. A. Rusinowska and H. De Swart. Generalizing and modifying the Hoede-Bakker index. In H. De Swart, E. Orlowska, G. Schmidt, and M. Roubens, editors, Theory and Applications of Relational Structures as Knowledge Instruments, LNAI 4342, pages 60-88, Heidelberg, 2006. Springer Verlag. 